\title{
Estimulação Elétrica Nervosa Transcutânea no Alívio da Dor Pós-Operatória Relacionada com Procedimentos Fisioterapêuticos em Pacientes Submetidos a Intervenções Cirúrgicas Abdominais* \\ Transcutaneous Electrical Nerve Stimulation in the Relief of Pain Related to Physical Therapy after Abdominal Surgery
}

\author{
Rodrigo Marques Tonella ${ }^{1}$, Sebastião Araújo², Áurea Maria Oliveira da Silva ${ }^{3}$
}

\section{RESUMO}

Tonella RM, Araújo S, Silva AMO - Estimulação Elétrica Nervosa Transcutânea no Alívio da Dor Pós-Operatória Relacionada com Procedimentos Fisioterapêuticos em Pacientes Submetidos a Intervenções Cirúrgicas Abdominais.

JUSTIFICATIVA E OBJETIVOS: A eletroestimulação nervosa transcutânea (TENS) tem sido pouco estudada como método facilitador da fisioterapia pós-operatória. O objetivo do estudo foi verificar a eficácia da TENS no alívio da dor relacionada com fisioterapia pós-operatória em pacientes submetidos a intervenções cirúrgicas abdominais.

MÉTODO: Foi realizado um estudo clínico, prospectivo, com distribuição aleatória, incluindo 48 pacientes no primeiro dia de pósoperatório, que apresentavam escore de dor $\geq 3$ pontos na escala visual analógica (VAS), divididos em três grupos: Grupo Controle: com rotina analgésica habitual, sem TENS e com fisioterapia; Grupo Estudo, recebendo rotina analgésica habitual mais TENS, e fisioterapia; Grupo Contraste-Placebo, recebendo rotina analgésica habitual, fisioterapia e TENS desligada. Foi apresentada aos pacientes uma escala visual analógica de dor antes (M1), depois da TENS (M2) e após fisioterapia (M3) - tosse, incentivador respiratório, mudança de decúbito lateral e sentado - quantificando a efetividade da analgesia. $O$ tempo de eletroestimulação foi 30 minutos.

RESULTADOS: Houve alívio significativo da dor no Grupo Estudo, comparando-se os três grupos, apenas para o procedimento tosse, no $M 3(p=0,015)$. Dentro desse grupo houve diminuição significativa da dor para tosse $(p=0,003)$ [M1 versus M3]; para decúbito lateral $(p=0,025)$, sentar-se $(p=0,001)$ e utilizar o incentivador inspiratório $(p=0,017)$ [M1 versus M2]; e ao se mudar para decúbito

${ }^{*}$ Recebido do (Received From) Enfermaria de Gastrocirurgia do Hospital das Clínicas da Universidade Estadual de Campinas (HC-Unicamp), Campinas, SP

1. Mestrando do Departamento de Cirurgia da FCM-UNICAMP; Fisioterapeuta do Centro de Terapia Intensiva da Unidade de Emergência do HC da FMRP-USP 2. Professor Assistente Doutor do Departamento de Cirurgia da FCM-UNICAMP 3. Doutoranda em Clínica Médica pela FCM-UNICAMP; Fisioterapeuta da Enfermaria de Gastrocirurgia do HC-UNICAMP

Apresentado (Submitted) em 26 de janeiro de 2006

Aceito (Accepted) para publicação em 30 de agosto de 2006

Endereço para correspondência (Correspondence to):

Dr. Rodrigo Marques Tonella

Rua Izabel Pantaleão Silveira, 304 - Parque São Quirino

13088-650 Campinas, SP

E-mail:digomato@terra.com.br

(c) Sociedade Brasileira de Anestesiologia, 2006 lateral $(p=0,03)$ e sentar-se $(p=0,001)[M 1 \times M 3]$. Não houve diferença significativa no Grupo Contraste-Placebo.

CONCLUSÕES: Houve diminuição da dor no Grupo Estudo, em alguns momentos e parâmetros. Estudos adicionais são necessários, já que a utilização da TENS está indicada apenas como coadjuvante no controle da dor pós-operatória.

Unitermos: ANALGESIA: estimulação elétrica nervosa tracutânea; CIRURGIA, Abdominal; DOR, Aguda: pós-operatória.

\section{SUMMARY}

Tonella RM, Araújo S, Silva AMO - Transcutaneous Electrical Nerve Stimulation in the Relief of Pain Related to Physical Therapy after Abdominal Surgery.

BACKGROUND AND OBJECTIVES: There are few studies on transcutaneous electrical stimulation (TENS) as an adjunct to postoperative physical therapy. The objective of this study was to determine the efficacy of TENS on relieving pain related to physical therapy after abdominal surgery.

METHODS: A clinical, randomized, prospective study was undertaken with 48 patients on the first postoperative day, who presented a pain score $\geq 3$ on the visual analogic scale (VAS). Patients were divided in three groups: Control Group: treated with the usual analgesic routine, without TENS, and with physical therapy; Study Group: treated with the usual analgesic routine associated with TENS and physical therapy; Contrast-Placebo Group: treated with the usual analgesic routine associated with physical therapy but TENS was off. A visual analogic scale of pain was presented to the patients before (M1), after TENS (M2), and after physical therapy (M3) - cough, incentive spirometry, changing lateral decubitus and sitting - to quantify the efficacy of the analgesia. Electrical stimulation was done during 30 minutes.

RESULTS: The Study Group presented a significant relief of the pain when compared to the other two groups only for coughing at $M 3$ ( $p=$ 0.015). In this group, there was a significant reduction in pain associated with coughing $(p=0.003)$ [M1 versus M3]; with lateral decubitus $(p=0.025)$, sitting ( $p=0.001)$, and with incentive spirometry ( $p=0.017$ ) [M1 versus M2]; and when changing to the lateral decubitus $(p=0.03)$ and sitting $(p=0.001)$ [M1xM3]. There were no significant differences in the Contrast-Placebo Group.

CONCLUSIONS: The Study Group presented a reduction in pain in a few moments and parameters. Further studies are needed since TENS is indicated only as an adjuvant in controlling postoperative pain.

Key Words: ANALGESIA: transcutaneous electric nerve stimulation; PAIN, Acute: postoperative; SURGERY, Abdominal. 
${ }^{\text {INTRO }}$ ODUÇÃo

inevitável que o procedimento cirúrgico cause lesão tecidual, seja pela manipulação cirúrgica direta visceral, ou através da própria incisão e pelo uso de afastadores cirúrgicos que auxiliam a exposição do campo operatório. $A$ presença de dor após a intervenção causa desconforto ao paciente, impedindo seu relaxamento, levando à ventilação superficial e prejudicando a movimentação no leito ${ }^{1}$. Dessa forma, complicações no pós-operatório são esperadas, decorrentes da dificuldade de equilíbrio entre as atividades muscular torácica e abdominal, deprimindo a respiração diafragmática e a efetividade da tosse, podendo redundar em atelectasias, sobretudo nos campos pulmonares inferiores, agravando ainda mais o processo inflamatório e a dor relacionados com o procedimento cirúrgico ${ }^{1}$.

Outro agravante e fator importante de risco para complicações pulmonares no pós-operatório é o tempo de duração do procedimento cirúrgico, que aumenta bastante as complicações pós-operatórias em função de tempo maior de exposição aos fatores de risco inerentes ao procedimento, como a ventilação mecânica, as medicações analgésicas e o acúmulo de secreções pulmonares ${ }^{2}$.

Diante disso, sabe-se que a movimentação e a deambulação precoces são essenciais para a recuperação efetiva e rápida no pós-operatório ${ }^{3}$. A deambulação, além de evitar complicações pulmonares, também previne o íleo paralítico, quase sempre presente no pós-operatório de operações abdominais ${ }^{4}$.

A dor é um fator de forte influência negativa na evolução pósoperatória do paciente submetido a procedimento cirúrgico abdominal, sobretudo àqueles da parte superior do abdômen, mesmo utilizando medicação analgésica.

A TENS é um recurso fisioterápico amplamente utilizado no alívio sintomático da dor. Ela é utilizada para estimular as fibras nervosas que transmitem sinais ao encéfalo, interpretados pelo tálamo como dor. Os impulsos transmitidos de forma transcutânea estimulam as fibras A, mielinizadas, transmissoras de informações ascendentes proprioceptivas. Essas fibras são sensíveis às ondas bifásicas e monofásicas interrompidas, como as da TENS. A base do efeito da TENS se dá conforme a "Teoria das Comportas", postulada por Melzack e Wall ${ }^{5}$, em 1965, e a superestimulação das fibras tipo A promove o bloqueio da entrada do estímulo pelas fibras tipo $C$ nas comportas do corno posterior da medula espinhal, na substância gelatinosa e nas células de transmissão (células T) ${ }^{6}$.

A TENS pode ser utilizada na rotina pós-operatória hospitalar como coadjuvante da analgesia convencional ${ }^{4,7,8}$. Além de ser não-invasiva e não-farmacológica, é confortável para o paciente em $95 \%$ dos casos ${ }^{4}$, pode ser eficiente no controle da dor pós-operatória abdominal ${ }^{9}$ e não possui efeitos colaterais associados ${ }^{3,8,9}$.

O uso da TENS pode auxiliar na prevenção de complicações pulmonares, como atelectasias, pneumonias e outras conseqüências decorrentes do acúmulo de secreções pulmo- nares, além de diminuir o uso de analgésicos ${ }^{10}$, aumentar mobilidade no leito e possibilitar a deambulação precoce. A presença de dor dificulta a atuação fisioterápica mais enérgica, no sentido de realizar manobras de higiene brônquica e exercícios precoces no leito ${ }^{9}$. A mobilização precoce do paciente no leito associada à deambulação são essenciais para sua rápida recuperação ${ }^{3,11}$.

O objetivo do estudo foi verificar a eficácia de forma complementar de analgesia - estimulação elétrica nervosa transcutânea (TENS) - com o intuito de diminuir o processo doloroso e reduzir o desconforto do paciente, permitindo uma atuação fisioterápica mais efetiva no pós-operatório.

\section{MÉTODO}

O protocolo de estudo foi aprovado pelo Comitê de Ética em Pesquisa da Instituição sob parecer no 141/2001. Foram incluídos no estudo 48 pacientes submetidos a intervenções cirúrgicas abdominais que apresentaram escore de dor $\geq 3$, medidos através da escala visual analógica (VAS) no primeiro dia de pós-operatório, com idade acima de 18 anos. O período de coleta de dados foi de 12 meses. Após informar aos pacientes todos os procedimentos a que seriam submetidos, foram obtidas as assinaturas do termo de consentimento livre e esclarecido.

O estudo foi prospectivo com distribuição aleatória dos pacientes, levando-se em conta a presença de dor com a intensidade descrita no primeiro dia de pós-operatório. Os pacientes foram divididos, por sorteio, em três grupos: Grupo Controle: obedeceu à rotina habitual de analgésicos do hospital e à fisioterapia; Grupo Estudo: obedeceu à rotina analgésica normal, acrescido da TENS, com fisioterapia de rotina e Grupo Contraste-Placebo: manteve-se a rotina analgésica, fisioterapia, aplicando-se a TENS, com o aparelho desligado.

Os critérios de exclusão foram pacientes com idade inferior a 18 anos, com deficiência cognitiva que impossibilitasse o entendimento e a assinatura do termo de consentimento para a participação no estudo, que necessitassem do uso de ventilação mecânica ou uso de fármacos vasoativos por instabilidade hemodinâmica no pós-operatório.

Foi utilizada uma unidade de eletroestimulação que contém a TENS, alimentada por tensão de rede elétrica, com eletrodos confeccionados com borracha condutora de silicone tratada com carbono, de formato retangular $(10 \times 3 \mathrm{~cm})$. Como meio de contato, utilizou-se gel aquoso, e a fixação dos eletrodos foi feita com esparadrapo antialérgico.

Para a coleta de dados utilizou-se uma ficha especialmente elaborada para este fim, contendo dados pessoais, tipo de intervenção cirúrgica, anestesia utilizada, tipo de incisão cirúrgica, tipo de analgesia pós-operatória utilizada, ausculta pulmonar e avaliação dos escores de dor para os procedimentos tosse, mudança voluntária de decúbito e realização do incentivador respiratório, em três momentos diferentes: antes da TENS (M1), logo após a TENS (M2) e após a aplicação da fisioterapia de rotina (M3). 
Para avaliação objetiva da dor foi usada a escala analógica visual graduada de 0 a 10, em que zero significava ausência de dor e 10, dor muito intensa. Ela serviu como parâmetro para a quantificação da dor, na medida em que foi apresentada ao paciente antes e após cada um dos procedimentos propostos para os três grupos, permitindo, assim, avaliar a alteração do escore de dor.

Os eletrodos foram dispostos de forma pericicatricial cruzada, a cerca de $2 \mathrm{~cm}$ da incisão cirúrgica (Figura 1). Não foi levado em consideração o tipo de incisão, mas sim a presença de dor com escore $\geq 3$ na VAS para pelo menos um procedimento fisioterápico e o primeiro dia de pósoperatório, para o uso da eletroestimulação. A duração do tratamento com a TENS foi de 30 minutos, e após cada um dos procedimentos avaliou-se novamente o escore da dor por meio da escala analógica visual. A fisioterapia respiratória consistiu na realização de manobras manuais de higiene brônquica, manobras manuais de reexpansão pulmonar, vibrocompressão com o auxílio de vibrador elétrico, aumento de fluxo expiratório e tosse assistida para auxiliar na expectoração de secreções pulmonares. Além da fisioterapia respiratória, realizou-se a fisioterapia motora incluindo a retirada do leito e deambulação com apoio, quando possível.

A freqüência utilizada para a TENS foi de $150 \mathrm{~Hz}$ com largura de pulso entre 150 a $250 \mu \mathrm{s}$, utilizando-se dois canais. A intensidade da estimulação foi modificada de acordo com a sensação relatada pelo paciente, com o intuito de promover estimulação intensa sem, no entanto, provocar desconforto. A rotina analgésica adotada pelo médico assistente foi mantida sem alterações.

Ao paciente, após ser recebido na enfermaria e posicionado no leito, foi apresentado ou lido o termo de consentimento livre e esclarecido. Após seu entendimento e esclarecimento de dúvidas, era solicitada sua assinatura ou impressão digital. Em seguida, com o paciente em repouso era apresentada a escala visual analógica de dor, explicando-lhe a

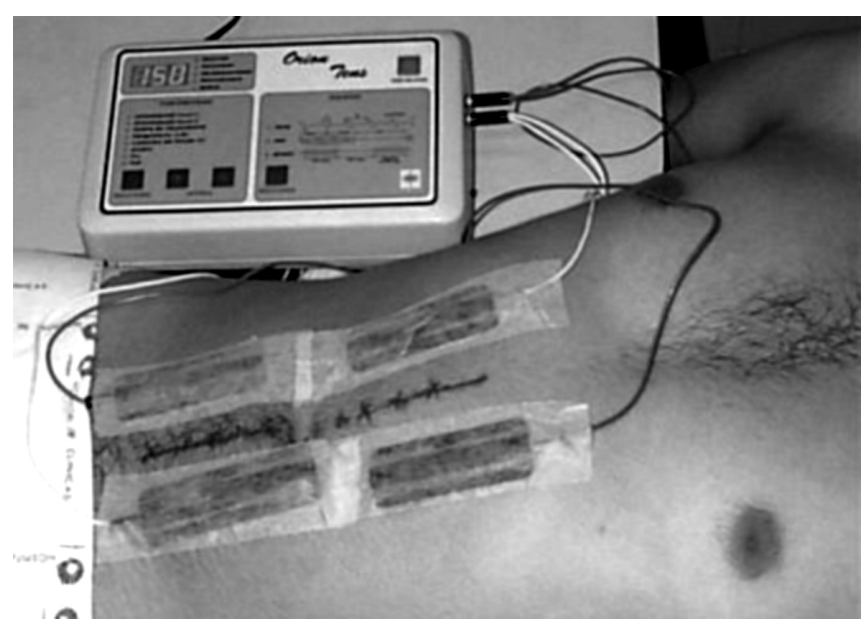

Figura 1 - Posicionamento Pericicatricial Cruzado dos Eletrodos. maneira correta de usá-la. Primeiro o paciente foi estimulado a tossir, na posição sentada, com inclinação do leito de aproximadamente 45우 em seguida, a fazer a mudança voluntária de decúbito, sentar-se e, por último, a realização do incentivador respiratório. Após cada procedimento, o escore da dor era registrado. Após a realização desses procedimentos, foi aplicada a TENS e os escores de dor novamente registrados ao final da eletroestimulação. Em um terceiro momento, foi realizada a fisioterapia respiratória e motora de rotina e, logo depois, foi aplicada a escala de dor para avaliar seu comportamento.

Para a avaliação de diferenças no escore de dor de acordo com os procedimentos fisioterápicos entre os três grupos foi utilizado o teste de Kruskal-Wallis. Para a verificação da existência de diferenças no escore de acordo com os procedimentos fisioterápicos dentro de cada grupo nos diferentes momentos (M1, M2 e M3) utilizou-se o teste de Friedman. Quando esse teste localizou alguma diferença entre os três momentos, utilizou-se o teste de Wilcoxon para identificar quais foram esses momentos (dois a dois). Para verificar se o fato do paciente ter utilizado ou não analgésico contribuiu para a existência de diferenças significativas foram aplicados os testes de Mann-Whitney e Wilcoxon. O nível significativo adotado foi de $5 \%$.

\section{RESULTADOS}

Foram avaliados neste estudo 48 pacientes, sendo 20 (42\%) do sexo feminino e 28 (58\%) do sexo masculino, com idade média de 49,6 anos no Grupo Controle, 54,1 anos no Grupo Estudo e 54 anos dentro no Grupo Contraste-Placebo. Os tipos de procedimentos cirúrgicos a que foram submetidos estão descritos na tabela I. Os tipos de incisões foram infraumbilical $(2 \%)$, subcostal direita $(17 \%)$, subcostal bilateral $(25 \%)$, supra-umbilical e infra-umbilical (17\%) e supra-umbilical (39\%). O padrão de analgesia farmacológica utilizada no pós-operatório antes da aplicação da TENS está descrito na tabela II.

$\mathrm{Na}$ comparação entre os grupos estudados com o objetivo de se detectar alguma diferença intergrupos, para cada parâmetro, em cada momento, ou seja, antes da TENS (M1), depois da TENS (M2) e depois da fisioterapia (M3), a única diferença estatística significativa foi encontrada no M3, ou seja, depois da TENS e da fisioterapia, para o parâmetro tosse $(p=0,015)$ (Figura 2).

Os escores de dor, dentro de cada grupo, comparando-se os diferentes momentos, também apresentaram diferenças estatísticas significativas (Figuras 3, 4 e 5).

Inicialmente foram demonstradas as diferenças dentro do Grupo Estudo, seguido pelos resultados do Grupo Controle. As comparações dentro do Grupo Contraste-Placebo não demonstraram diferenças estatísticas significativas em nenhum momento ou parâmetros avaliados.

Ainda dentro do Grupo Estudo, houve diferença significativa para os parâmetros sentar-se e mudar de decúbito, em di- 
ferentes momentos, como demonstrado na tabela III. A diminuição do escore de dor foi significativa para sentar-se (M1 versus $\mathrm{M} 2$ e M1 versus $\mathrm{M} 3$ ) e também durante a mudança de decúbito (M1 versus M2 e M2 versus M3).

Tabela I - Procedimentos Cirúrgicos Realizados

\begin{tabular}{lcc}
\hline & Freqüência & Porcentual \\
\hline Anastomose hepatojejunal & 2 & 4 \\
Colecistectomia & 7 & 14 \\
Relaparotomia & 1 & 2 \\
Derivação bileodigestiva & 4 & 8 \\
Gastroenterectomia & 1 & 2 \\
Gastroplastia & 4 & 8 \\
Gastrectomia & 11 & 23 \\
Gastroduodenopancreatectomia & 1 & 2 \\
Gastrostomia & 1 & 2 \\
Degastrectomia & 1 & 2 \\
Derivação pancreatojejunal & 1 & 2 \\
Esofagogastrectomia & 2 & 4 \\
Hepatectomia & 3 & 6 \\
Enteroanastomose & 1 & 2 \\
Esplenectomia & 2 & 4 \\
Gastrectomia/deriv. Pancreatojejunal & 3 & 6 \\
Laparotomia & 2 & 4 \\
Pancreatoesplenocolecistectomia & 1 & 2 \\
\hline & & \\
\hline
\end{tabular}

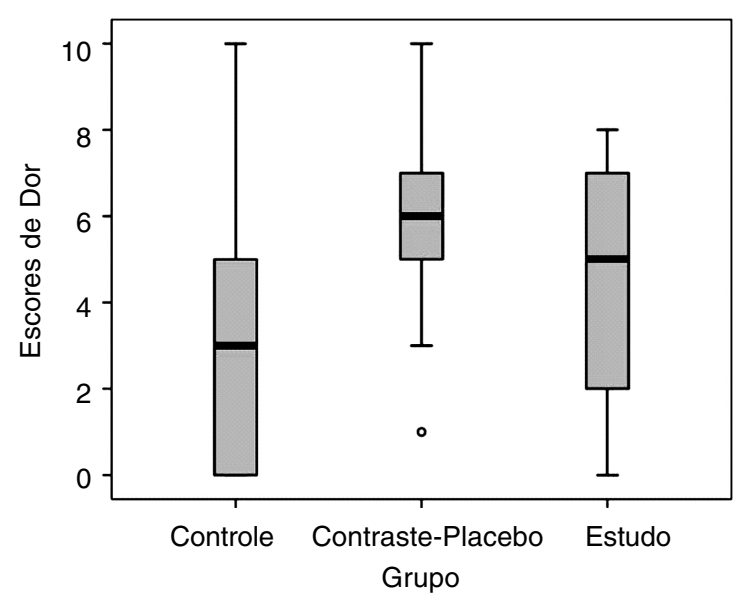

Figura 2 - Escores de Dor entre os Três Grupos Estudados. O único valor estatístico significativo do Grupo Estudo com relação aos demais grupos foi no M3 (depois da TENS e da fisioterapia), para o procedimento tosse, com diminuição do escore de dor $(p=0,015)$.
Tabela II - Analgesia Utilizada no Pós-Operatório de Procedimentos Cirúrgicos Abdominais

\begin{tabular}{lcc}
\hline & Freqüência & Percentual \\
\hline Nenhum* & 16 & 34 \\
Morfina & 4 & 8 \\
Morfina/dipirona & 2 & 4 \\
Morfina/tenoxicam & 3 & 38 \\
Morfina/tramadol/tenoxicam & 1 & 6 \\
Tenoxicam/dipirona & 2 & 4 \\
Tenoxicam & 18 & 2 \\
Tenoxicam/dipirona/adifenina/ & 1 & 2 \\
prometazina & & \\
Tramadol & 1 & 2 \\
\hline
\end{tabular}

*Pacientes que estavam com prescrição de medicação analgésica de rotina, mas que foram submetidos à eletroestimulação antes da administração do analgésico naquele horário

Tabela III - Valores de p para as Variáveis Sentar-se e Mudança de Decúbito, dentro do Grupo Estudo

\begin{tabular}{llcc}
$\begin{array}{l}\text { Grupo Estudo } \\
\text { Parâmetro }\end{array}$ & \multicolumn{2}{c}{ Momentos } & $p$ \\
\hline Sentado & M1 & M2 & 0,001 \\
Sentado & M1 & M3 & 0,001 \\
Decúbito lateral & M1 & M2 & 0,025 \\
Decúbito lateral & M2 & M3 & 0.03
\end{tabular}

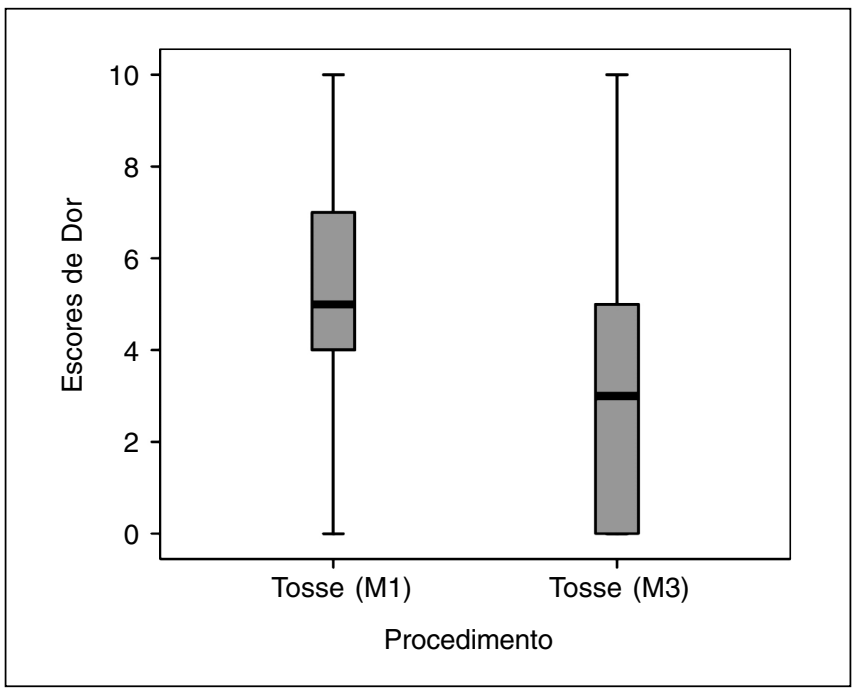

Figura 3 - Escores de Dor entre os Momentos e Procedimentos no Grupo Estudo. Houve diferença estatística significativa entre os escores de dor no M1 versus M3, ao se realizar a tosse ( $p=$ 0,009 ), com diminuição do escore de dor ao realizar esse procedimento, depois da eletroestimulação e do procedimento fisioterapêutico (M3). 


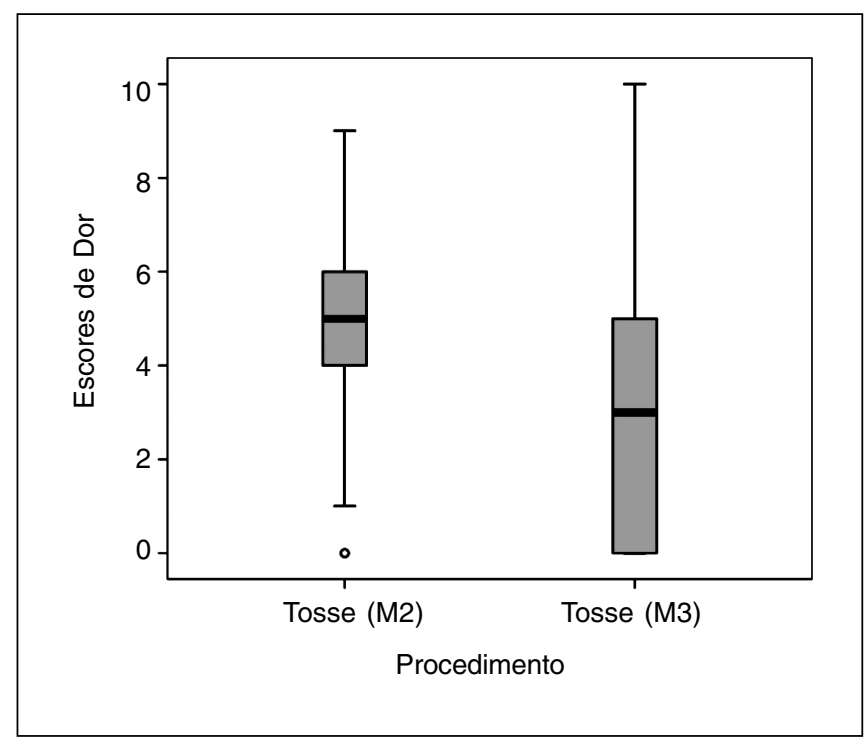

Figura 4 - Escores de Dor entre os Momentos e Procedimentos no Grupo Estudo. Houve diferença estatística significativa entre os escores de dor no $M 2$ versus $M 3$, ao se realizar a tosse ( $p=$ $0,003)$, com diminuição do escore de dor ao realizar esse procedimento, depois da eletroestimulação e do procedimento fisioterapêutico (M3).

\section{DISCUSSÃO}

A dor pós-operatória é uma questão que envolve e preocupa a equipe multiprofissional, no sentido de escolher a meIhor forma de diminuí-la, sobretudo pelo fato de que sua presença pode mascarar complicações cirúrgicas pós-operatórias ${ }^{12} \mathrm{e}$ dificultar a realização da fisioterapia. A dor incisional pós-operatória em repouso e durante a movimentação é uma das formas de manifestação dos processos de lesão celular e de inflamação decorrentes do ato cirúrgico e que, muitas vezes, é difícil de ser controlada por meio da analgesia convencional com opióides ${ }^{13}$. Nguyen e col. ${ }^{14}$, em 2001, concluíram que a gastroplastia, por via laparoscópica, provocou menos dor durante o repouso e a mobilização do paciente comparada com a técnica tradicional, além de promover diminuição no uso de morfina no pós-operatório ( $p<0,001)$, fato este possivelmente secundário a menor grau de lesão cirúrgica. Neste estudo, os pacientes no pós-operatório imediato, ao serem indagados sobre a dor, referiram a sua presença na incisão cirúrgica na maioria dos casos estudados.

A analgesia medicamentosa pós-operatória de rotina foi mantida em todos os pacientes do estudo. No entanto, alguns foram assistidos pelo fisioterapeuta e avaliados no protocolo, antes da administração da analgesia daquele horário. A análise estatística realizada mostrou que não houve influência da presença ou ausência da medicação analgésica, dentro dos três grupos, na maioria dos procedimentos e momentos. A fisioterapia pós-operatória é um procedimento necessário na recuperação do paciente, atuando de forma preventiva e curativa em algumas possíveis complicações, como

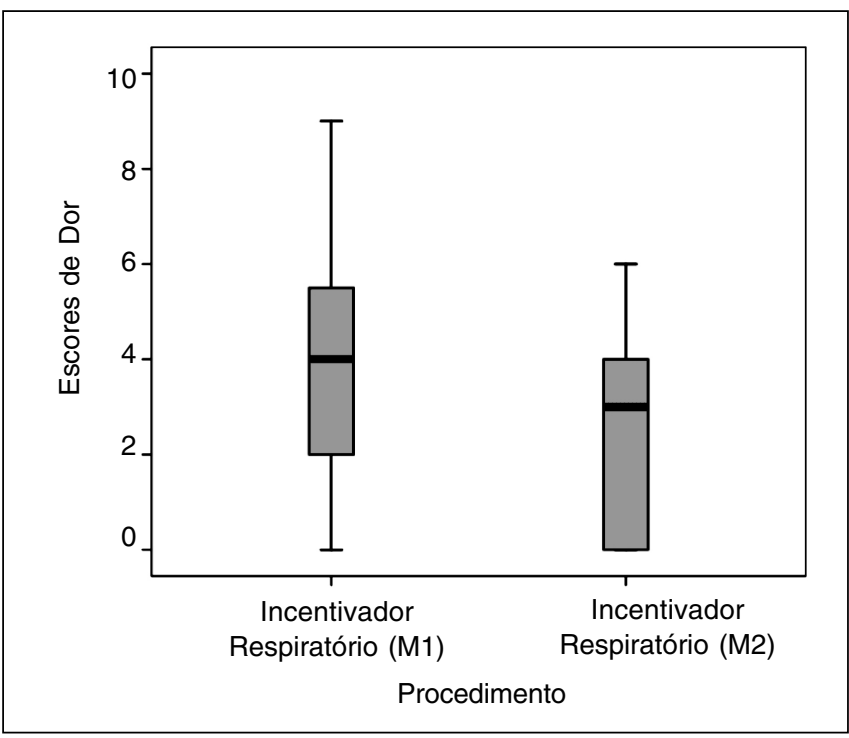

Figura 5 - Escores de Dor entre os Momentos e Procedimentos no Grupo Estudo. Houve diferença estatística significativa entre o escore de dor no M1 versus $\mathrm{M} 2$, ao se realizar o incentivador respiratório $(p=0,017)$, com diminuição do escore de dor ao realizar esse procedimento, depois da eletroestimulação (M2).

atelectasias e pneumonia ${ }^{1}$. Os procedimentos fisioterápicos de rotina podem potencializar o processo doloroso do paciente ${ }^{15}$, pois utilizam recursos manuais e exercícios específicos aliados à mobilização intensa do paciente, promovendo desde melhora da função respiratória (manutenção da permeabilidade e expansão pulmonar) até a possibilidade de deambulação mais precoce (reabilitação motora). A fisioterapia respiratória pós-operatória consiste em manobras manuais e mecânicas (com uso de vibrador elétrico) de reexpansão pulmonar, mobilização de secreções através de vibrocompressão torácica manual, estímulo de tosse por comando verbal, técnica de tosse assistida, estímulo de fúrcula esternal e estímulo à reexpansão pulmonar por meio de exercícios respiratórios e utilização de incentivadores respiratórios ${ }^{16}$. A tosse forçada, para o paciente no pós-operatório, sobretudo e após intervenção cirúrgica de abdômen superior, é um dos procedimentos que geram mais dor, pois exige a contração vigorosa da musculatura abdominal e do diafragma na presença de uma incisão cirúrgica local que está em franco processo inflamatório e doloroso. Embora seja um dos procedimentos que mais levará a aumento da dor, a tosse é essencial para o deslocamento e a expectoração da secreção pulmonar acumulada, garantindo a proteção do sistema respiratório, sua higiene e equilíbrio, diminuindo assim o risco de atelectasias e infecções. A TENS é um recurso fisioterápico que pode ser usado como coadjuvante da analgesia convencional ${ }^{4,7,8,13}$, sendo nãoinvasiva e virtualmente sem efeitos colaterais importantes. A utilização dessa forma de analgesia foi eleita no presente estudo para promover o alívio da dor pós-operatória, so- 
bretudo a dor causada pelo procedimento fisioterápico, em pacientes no pós-operatório de procedimentos cirúrgicos em abdômen superior, pois, quanto maior for a colaboração e sua participação na execução do tratamento com exercícios, melhor será a efetividade da técnica e mais rápida será a recuperação e reabilitação do paciente.

Bjordal e col. ${ }^{10}$, recentemente, publicaram metanálise dos trabalhos que utilizaram a TENS como recurso analgésico no pós-operatório, compreendendo o período entre 1966 e 2001. Os resultados demonstraram que a TENS reduziu 0 consumo de medicação analgésica durante os três primeiros dias de $\mathrm{PO}$, diminuindo os efeitos colaterais da medicação opióide administrada, além de promover redução com diferença estatística significativa dos escores de dor no Grupo Estudo, comparados com o Grupo Contraste-Placebo, fato que também foi demonstrado no presente trabalho. Outra importante conclusão desses autores é o fato de em geral se necessitar de intensidade de corrente elevada (o máximo tolerado pelo paciente, sem causar desconforto) para maior efetividade da eletroanalgesia, o que vem de encontro ao método utilizado na presente pesquisa.

A dor pós-operatória é causada basicamente pela lesão do tecido e o subseqüente processo inflamatório. Aliada a essa causa inicial, existe a hiperalgesia primária pós-operatória, que seria basicamente a extensão do processo doloroso local para regiões adjacentes à lesão, em função da intensa liberação de mediadores algogênicos, responsáveis por potencializar o processo doloroso original. Esse processo leva a redução do limiar doloroso e a aumento da sensibilidade das terminações nervosas adjacentes, causando hiperalgesia a estímulos que antes não geravam dor, como o estímulo tátil, por exemplo ${ }^{17}$. A realização de qualquer procedimento durante a vigência desse processo pode gerar ainda mais dor, sobretudo durante a mobilização e movimentação do paciente que provoca mobilização da região incisional ${ }^{13}$.

A TENS foi eficaz no alívio da dor provocada pelo procedimento fisioterápico, nesta pesquisa, comparando-se todos os grupos, em todos os momentos, apenas no parâmetro tosse no $M 3$, ou seja, após a fisioterapia $(p=0,015)$. A importância da diminuição do escore de dor para realização da tosse é relevante, frente às possíveis complicações pulmonares que podem ocorrer no pós-operatório de procedimentos cirúrgicos abdominais. Além disso, a tosse sendo realizada com menos dor, pode significar a garantia de higiene brônquica efetiva, reabilitação pulmonar precoce e prevenção de atelectasias e pneumonias secundárias ao acúmulo de secreções pulmonares. Este dado coincide com o resultado de Ali e col. ${ }^{9}$, que demonstraram também a eficácia da TENS no alívio da dor pós-operatória, levando-se em conta o aumento da capacidade vital (CV) e da capacidade residual funcional (CRF), diminuindo a tendência a complicações respiratórias em virtude do alívio da dor incisional.

Além desse importante dado, dentro do grupo TENS também houve redução nos escores de dor ao se realizar al- guns procedimentos antes e após a eletroestimulação e após a fisioterapia, evidenciando a importância da terapêutica com a TENS como coadjuvante para a realização da fisioterapia respiratória e motora.

A tosse gerou um escore de dor menor nos pacientes pertencentes ao Grupo Estudo, comparando-se o escore do M1 com o do M3 $(p=0,009)$ e comparando-se o escore do M2 com o do $M 3(p=0,003)$. Essa diminuição dos escores de dor demonstrou a efetividade da TENS como coadjuvante no controle da dor para realização da fisioterapia respiratória. É importante ressaltar que o escore do M3 (após a fisioterapia) foi ainda menor que os escores do M1 e M2. A efetividade da analgesia com TENS foi importante para a realização do procedimento fisioterápico necessário, e o decréscimo do escore no último momento denota a continuidade do efeito analgésico conseguido com a TENS na realização da tosse. Esse efeito conseguido após fisioterapia pode ser explicado pela modulação da dor realizada pela TENS nas fibras condutoras de estímulos nociceptivos denominadas A delta. Durante a movimentação, a dor desencadeada é transmitida pela fibra A delta. No entanto, a ação da medicação opióide acontece de forma inibitória para a fibra $\mathrm{C}$, tornando-a pouco efetiva no controle da dor desencadeada pela movimentação ou tosse ${ }^{12,13}$. A TENS gera um estímulo tátil que é transmitido através da fibra $\mathrm{C}$, bloqueando a passagem do estímulo doloroso gerado pela fibra A delta ${ }^{13}$.

Além da fisioterapia respiratória realizada, os pacientes foram submetidos a exercícios ativos de membros superiores e inferiores, foram auxiliados a sentar-se fora do leito e, mesmo após todos esses procedimentos, o escore final ao se realizar a tosse ainda decresceu.

A utilização de incentivadores respiratórios no pós-operatório de procedimentos cirúrgicos abdominais é preconizada como técnica auxiliar na reexpansão pulmonar, pois fornece ao paciente o feedback da sua realização através do número de esferas elevadas a cada inspiração, e é amplamente usada no ambiente hospitalar como forma profilática das complicações pulmonares ${ }^{18}$. A correta utilização desse dispositivo depende de um entendimento da técnica correta, para seu perfeito desempenho. A realização desse exercício necessita de uma reeducação diafragmática, no sentido de promover uma expansão pulmonar de preferência em bases, com um padrão respiratório predominantemente diafragmático e com inspiração máxima sustentada ${ }^{19}$. A excursão diafragmática está debilitada pelo processo doloroso e pelo edema local provocado pelo procedimento cirúrgico abdominal, sendo a aplicação do incentivador potencial fonte adicional de dor.

Houve diminuição significativa dos escores de dor para a realização do incentivador respiratório no Grupo Estudo, quando se comparou o M1 com o M2. A TENS foi efetiva no alívio da dor para a realização do incentivador apenas no segundo momento (M2), ou seja, logo após a eletroestimulação. Nesse parâmetro, não houve diminuição do escore de dor 
no $M 3$, após a fisioterapia, não se conseguindo demonstrar a efetividade analgésica da TENS no controle da dor para essa modalidade no M3. Como o aprendizado da técnica pode influenciar diretamente na efetividade em realizar o incentivador respiratório, é possível que os pacientes já estivessem familiarizados com o dispositivo e/ou estivessem com um padrão ventilatório melhor após a fisioterapia respiratória, sentados fora do leito, o que os levou a realizarem o incentivador com maior efetividade, resultando assim num escore de dor maior do que aquele registrado no segundo momento, logo após a TENS.

A diminuição dos escores de dor, dentro dos momentos no Grupo Estudo, aconteceu também durante os procedimentos mudança de decúbito e posicionamento sentado. Houve diferenças estatísticas significativas entre o M1 versus M2 ( $p=0,025)$ e entre o M1 versus M3 $(p=0,03)$ para mudar de decúbito, e entre $M 1$ versus $M 2(p=0,001)$ e $M 1$ versus $M 3$ ( $p=0,001$ ) para sentar-se. É importante salientar que nesses procedimentos a efetividade da TENS foi evidenciada já no M2, ou seja, logo após sua aplicação. O seu efeito se prolongou também após a realização da fisioterapia (respiratória e motora), demonstrando um escore ainda menor que o inicial (antes da TENS). É importante ressaltar a diminuição do escore para a realização da mudança de decúbito e da posição sentada, pois esses procedimentos exigem o recrutamento intenso da musculatura abdominal (retos abdominais e oblíquos) para produzir o movimento e essa contração muscular acontece na vigência de uma incisão abdominal que provoca dor quando mobilizada ${ }^{13}$. A movimentação precoce do paciente é importante, pois previne o íleo adinâmico ${ }^{4}$, as complicações respiratórias já descritas e até mesmo complicações vasculares, como a trombose venosa profunda 20

A deambulação foi inicialmente escolhida como parâmetro de avaliação do escore de dor, mas a sua aplicação foi muito comprometida, pois era necessária a realização desse procedimento antes de qualquer outra intervenção, dentro dos três momentos, sendo a deambulação geralmente feita após a realização de todo procedimento de fisioterapia respiratória e motora no leito, impedindo a inclusão desse parâmetro de avaliação no estudo, embora tenha sido realizado, dentro da rotina de fisioterapia motora, no M3.

Outro fator a ressaltar é que na literatura há poucos trabalhos avaliando a aplicação da TENS com o objetivo de aliviar a dor durante a realização de fisioterapia. A maioria dos trabalhos compara a analgesia com TENS e a convencional ou a diminuição da demanda de analgésico com a aplicação da TENS, sem levar em conta a necessidade do paciente realizar exercícios fisioterápicos pós-operatórios. Dentro dessa linha, e em acordo com os resultados desse estudo, Rakel e Frantz ${ }^{13}$, em 2003, demonstraram que a TENS reduziu a intensidade da dor para a realização de inspiração profunda e para realização de caminhada no primeiro dia pós-operatório.

A utilização da TENS levou à diminuição dos escores de dor para alguns procedimentos e momentos das intervenções fisioterápicas. No entanto, esses resultados devem ser analisados com cautela, pois a casuística foi pequena e as diferenças não foram grandes. Assim, a aplicação da TENS como alternativa analgésica não-farmacológica deve ser mais bem estudada, já que a sua utilização ainda é indicada apenas como coadjuvante no controle da dor pós-operatória e da dor provocada pelo procedimento fisioterápico.

\section{AGRADECIMENTOS}

À colaboradora fisioterapeuta Luciana Ysayama, mestre em Pesquisa Experimental pelo Departamento de Cirurgia da Faculdade de Ciências Médicas da Universidade Estadual de Campinas (FCM-UNICAMP), e ao Professor José Milton Sanches pela assessoria na análise estatística dos dados.

\section{Transcutaneous Electrical Nerve Stimu- lation in the Relief of Pain Related to Phy- sical Therapy after Abdominal Surgery}

Rodrigo Marques Tonella, M.D.; Sebastião Araújo, M.D.; Áurea Maria Oliveira da Silva, M.D.

\section{INTRODUCTION}

Surgical procedures cause, inevitably, tissue damage, may it be by direct visceral manipulation, through the incision itself, and by the use of surgical retractors that help expose the surgical field. Pain after surgery causes discomfort to the patient, preventing the patient from relaxing, leading to shallow breathing, and hindering the patient's movements in bed ${ }^{1}$. Thus, postoperative complications secondary to a difficult balance between thoracic and abdominal muscular activities, which decrease diaphragmatic breathing and cough efficacy that might lead to atelectasis, especially in the lower lung fields, are expected to worsen the inflammatory process and pain related to the surgical procedure even more ${ }^{1}$.

The duration of the surgical procedure is another aggravating and important risk factor for postoperative pulmonary complications. It increases significantly postoperative complications due to the greater exposure to risk factors inherent to the procedure, such as mechanical ventilation, analgesics, and the accumulation of pulmonary secretions ${ }^{2}$.

Moreover, it is known that early movement and ambulation are fundamental for an effective and fast postoperative recovery ${ }^{3}$. Besides preventing pulmonary complications, ambulation also prevents the ileus that is frequent in the postoperative period of abdominal surgeries ${ }^{4}$.

Pain is an important negative influence in the postoperative evolution of abdominal surgeries, especially those in the upper abdomen, even using analgesic drugs.

Transcutaneous electrical stimulation is a physical therapy tool widely used to relieve pain. It stimulates nerve fibers that send signals to the brain, which the thalamus interprets as 
pain. The impulses transmitted transcutaneously stimulate myelinated A fibers, which transmit proprioceptive ascendant information. These fibers are sensitive to interrupted biphasic and monophasic waves, such as those delivered by TENS. The effects of TENS follow the "Gate Theory" postulated by Melzack et al. ${ }^{5}$ in 1965, in which the superstimulation of type A fibers block the entrance of stimuli conducted by type $C$ fibers in the gates of the gelatinous substance, the posterior horn of the spinal cord, and the transmission cells ${ }^{6}$. Transcutaneous electrical stimulation can be used in the postoperative hospital routine as adjuvant to conventional analgesia ${ }^{4,7,8}$. Besides being a non-invasive and non-pharmacologic technique, it is comfortable for the patient in $95 \%$ of the cases ${ }^{4}$, can be effective in controlling pain after abdominal surgeries ${ }^{9}$, and has no side effects ${ }^{3,8,9}$.

It can prevent pulmonary complications, such as atelectasis, pneumonias, and others, secondary to the accumulation of pulmonary secretions, besides decreasing the use of analgesics ${ }^{10}$, increasing mobility in bed, and allowing for early ambulation. Pain hinders the use of more vigorous physical therapy for bronchial hygiene and early exercises in bed ${ }^{9}$. Early movement in bed associated with ambulation is fundamental for the patient's speedy recovery ${ }^{3,11}$.

The aim of this study was to determine the efficacy of transcutaneous electrical stimulation (TENS) as an adjunct in decreasing patient's pain and discomfort, allowing for more effective physical therapy maneuvers in the postoperative period.

\section{METHODS}

This study was approved by the Ethics Committee on Research of the Institution, protocol number 141/2001. Fortyeight patients, older than 18 , who underwent abdominal surgeries and presented a pain score $\geq 3$, measured by the visual analogic scale (VAS) on the first postoperative day, were included in this study. Data were gathered during a 12month period. After informing patients about the procedures to which they would be submitted, an informed consent was signed.

This was a randomized, prospective study, taking into consideration the presence of pain in the same severity reported in the first postoperative day. Patients were randomly divided in three groups: Control Group: followed the usual hospital analgesic routine and physical therapy; Study Group: followed the usual hospital routine regarding analgesics associated with TENS and physical therapy; and Contrast-Placebo Group: followed the analgesic routine, TENS was applied, but it was not turned on.

Exclusion criteria included patients younger than 18 years, with cognitive deficiencies that hinder understanding and signing of the informed consent, and the need for mechanical ventilation or vasoactive drugs for postoperative hemodynamic instability.

An electrical stimulation unit containing TENS, connected to the electrical network, and with rectangular $(10 \times 3 \mathrm{~cm})$ siliconerubber, carbon-treated electrodes. An aqueous gel was used and electrodes were fixed to the skin with hypoallergenic tape. A form designed especially for this study was used to record the data, which included personal data, type of surgery, anesthesia, type of surgical incision, type of postoperative analgesia, pulmonary auscultation, and pain scores for cough, voluntary change of decubitus, and use of incentive spirometry in three different moments: before TENS (M1), immediately after TENS (M2), and after routine physical therapy (M3). The visual analogic scale, graded from zero to 10 , in which zero means no pain, and 10 very severe pain, was used for the objective evaluation of pain. It was used as a parameter to quantify pain, and was shown to the patient before and after each procedure proposed for the three groups allowing, therefore, for an evaluation of the change in pain score.

Electrodes were placed in a crossover periscar distribution, about $2 \mathrm{~cm}$ from the surgical wound (Figure 1). To apply TENS, the type of incision was not taken into consideration, but the presence of a pain score $\geq 3$ in the VAS for at least one physical therapy procedure and the first postoperative day were considered. Treatment with TENS lasted 30 minutes, and after each procedure the pain score was evaluated once more using the visual analogic scale. Respiratory physical therapy consisted of manual maneuvers of bronchial toilette, manual maneuvers of pulmonary reexpansion, vibro-compression using an electric vibrator, increase in the expiratory flow, and assisted coughing to aid expectoration of pulmonary secretions. Besides respiratory physical therapy, motor physical therapy, including leaving the bed and assisted ambulation, when possible, was also done.

The frequency used for TENS was $150 \mathrm{~Hz}$ with a pulse width between 150 and $250 \mu$ s, using two channels. The intensity of the electrical stimulation was changed according to the feeling reported by the patient in order to promote intense stimulation without discomfort. The physician's analgesic prescription was not changed.

After the patient arrived in the ward and was placed in bed, the informed consent was read to him/her. After all questions

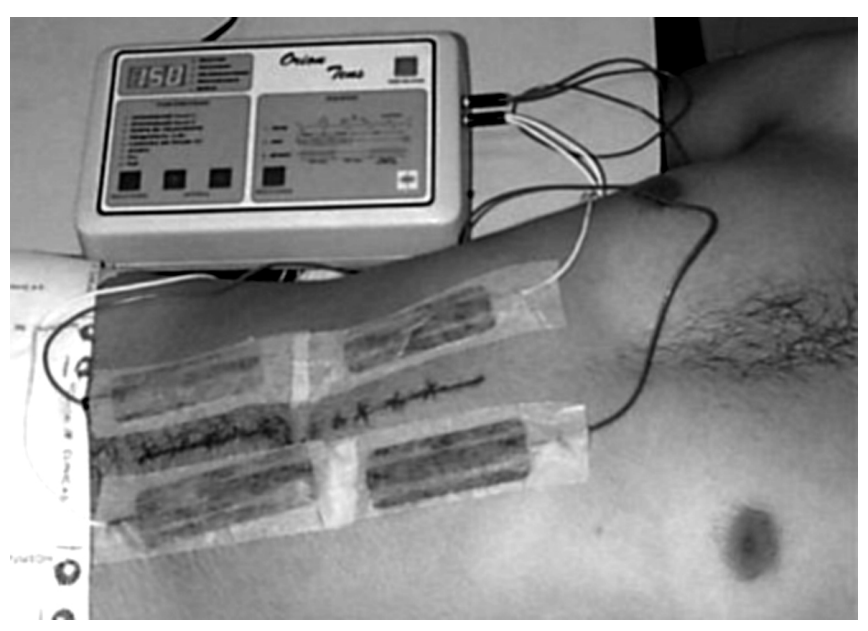

Figure 1 - Crossover Periscar Placement of Electrodes. 
were answered, he/she signed the form or was asked to place his/her fingerprint. The visual analogic pain scale was shown to the patient at rest, explaining the proper way of using it. He was then stimulated to cough, while in the sitting position, with the bed at an inclination of about $45^{\circ}$. It was then followed by a voluntary change in decubitus, sitting, and finally the incentive spirometry. Pain scores were recorded after each procedure. Transcutaneous electrical stimulation was applied after these procedures after which pain scores were recorded once again. Respiratory and routine motor physical therapy were then performed and pain scores were recorded again.

The Kruskal-Wallis test was used to evaluate the differences in pain scores according to the physical therapy procedure among the three groups. The Friedman test was used to determine the presence of score differences according to the physical therapy procedures within each group at different moments (M1, M2, and M3). When this test demonstrated any difference, the Wilcoxon test was used to identify those moments (two by two). The Mann-Whitney and Wilcoxon tests were used to determine whether the use of analgesics contributed for the significant differences. A $5 \%$ level was considered significant.

\section{RESULTS}

Forty-eight patients, 20 (42\%) women and $28(58 \%)$ men, mean age of 49.6 years in the Control Group, 54.1 years in the Study Group, and 54 years in the Contrast-Placebo Group, were evaluated in this study. Table I shows the types of surgical procedures patients underwent. Incisions were infra umbilical $(2 \%)$, right subcostal $(17 \%)$, bilateral subcostal (25\%), supra- and infra-umbilical (17\%), and supra umbilical $(39 \%)$. Table II shows the postoperative pharmacological analgesia used before TENS.

Comparing study groups in order to detect any intergroup differences for each parameter in each moment, i.e., before TENS (M1), after TENS (M2), and after physical therapy (M3), the only statistically significant difference occurred for the parameter cough in $\mathrm{M} 3$, that is, after TENS and physical therapy $(p=0.015)$ (Figure 2).

Pain scores within each group at the different moments also showed statistically significant differences (Figures 3 , 4 , and 5).

Initially, the differences within the Study Group, followed by the results of the Control Group are shown. The comparisons within the Contrast-Placebo Group did not show statistically significant differences at any moment for any of the parameters.

In the Study Group there were also differences for the parameters sitting and decubitus change in different moments, as shown in Table III. Pain scores were significantly reduced for the parameter sitting (M1 versus $\mathrm{M} 2$ and $M 1$ versus $M 3$ ), and also during changes in decubitus (M1 versus $\mathrm{M} 2$ and $\mathrm{M} 2$ versus $\mathrm{M} 3$ ).
Table I - Types of Surgeries

\begin{tabular}{lcc}
\hline & Frequency & Percentage \\
\hline Hepatojejunal anastomosis & 2 & 4 \\
Cholecystectomy & 7 & 14 \\
Repeated laparotomy & 1 & 2 \\
Biliary-digestive bypass & 4 & 8 \\
Gastroenterectomy & 1 & 2 \\
Gastroplasty & 4 & 8 \\
Gastrectomy & 11 & 23 \\
Gastroduodenopancreatectomy & 1 & 2 \\
Gastrostomy & 1 & 2 \\
Degastrectomy & 1 & 2 \\
Pancreato-jejunal bypass & 1 & 2 \\
Esophaogastrectomy & 2 & 4 \\
Hepatectomy & 3 & 6 \\
Enteroanastomosis & 1 & 2 \\
Splenectomy & 2 & 4 \\
Gastrectomy/pancreato-jejunal bypass & 3 & 6 \\
Laparotomy & 2 & 4 \\
Pancreatosplenocolecystectomy & 1 & 2 \\
\hline
\end{tabular}

Table II - Analgesics Used after Abdominal Surgeries

\begin{tabular}{lcc}
\hline & Frequency & Percentage \\
\hline None* & 16 & 34 \\
Morphine & 4 & 8 \\
Morphine/dypirone & 2 & 4 \\
Morphine/tenoxicam & 3 & 38 \\
Morphine/ tramadol/tenoxicam & 1 & 6 \\
Tenoxicam/dypirone & 2 & 4 \\
Tenoxicam & 18 & 2 \\
Tenoxicam/dypirone/adifenine/prometazine & 1 & 2 \\
Tramadol & 1 & 2 \\
\hline
\end{tabular}

*Patients who had a routine analgesic therapy prescribed but had TENS before the scheduled administration of the analgesic

Table III - Values of $p$ for Sitting and Decubitus Change in the Study Group

\begin{tabular}{lllc}
\hline $\begin{array}{l}\text { Study Group } \\
\text { Parameter }\end{array}$ & \multicolumn{2}{c}{ Moments } & $\mathrm{p}$ \\
\hline Sitting & M1 & M2 & 0.001 \\
Sitting & M1 & M3 & 0.001 \\
Lateral decubitus & M1 & M2 & 0.025 \\
Lateral decubitus & M2 & M3 & 0.03 \\
\hline
\end{tabular}




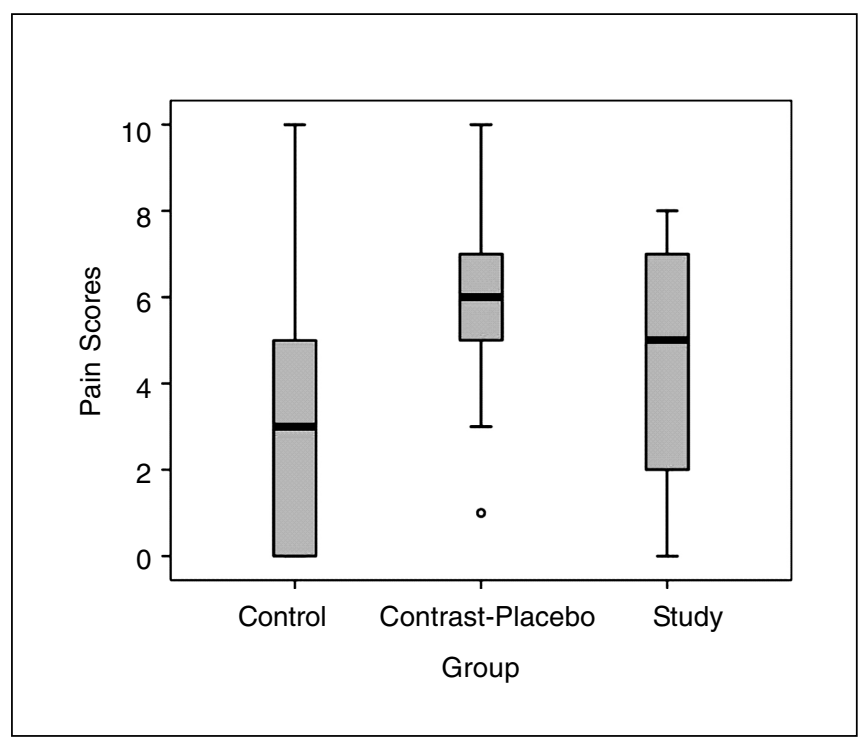

Figure 2 - Pain Scores among The Three Study Groups. The only value statistically significant in the Study Group when compared to the other groups (after TENS and after physical therapy) was $\mathrm{M} 3$ for coughing, with a reduction in pain scores $(p=0.015)$.

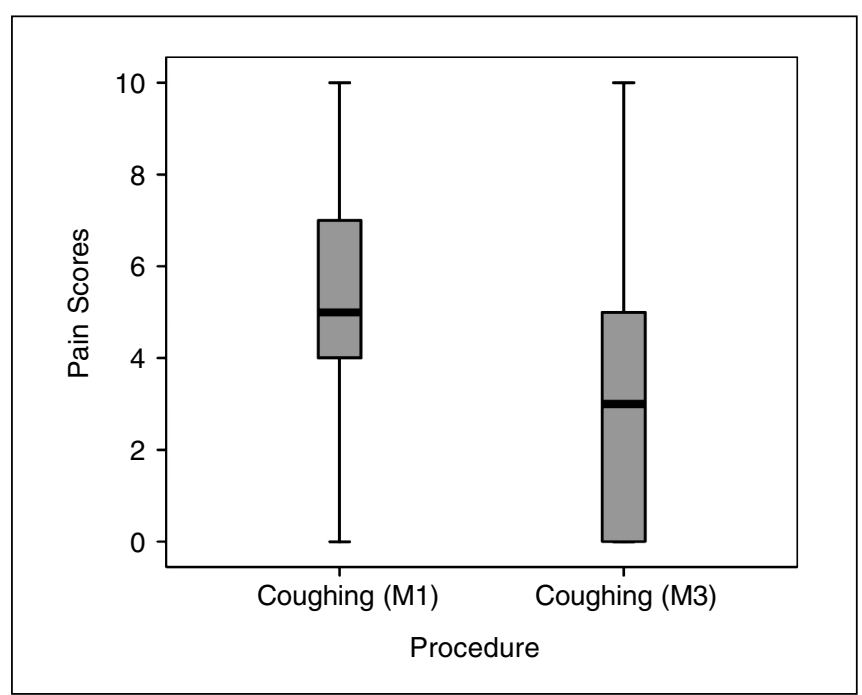

Figure 3 - Pain Scores among Moments and Procedures in the Study Group. There was a statistically significant difference in pain scores between M1 and M3 for coughing ( $p=0.009)$, with a reduction in pain score for this maneuver, after electrical stimulation and physical therapy (M3).

\section{DISCUSSION}

Postoperative pain involves and worries the multidisciplinary team as far as choosing the best way of decreasing it, especially because its presence can mask postoperative surgical complications ${ }^{12}$ and hinder physical therapy. Incisional pain at rest and during movements is one way that cellular lesion and inflammation secondary to the surgery

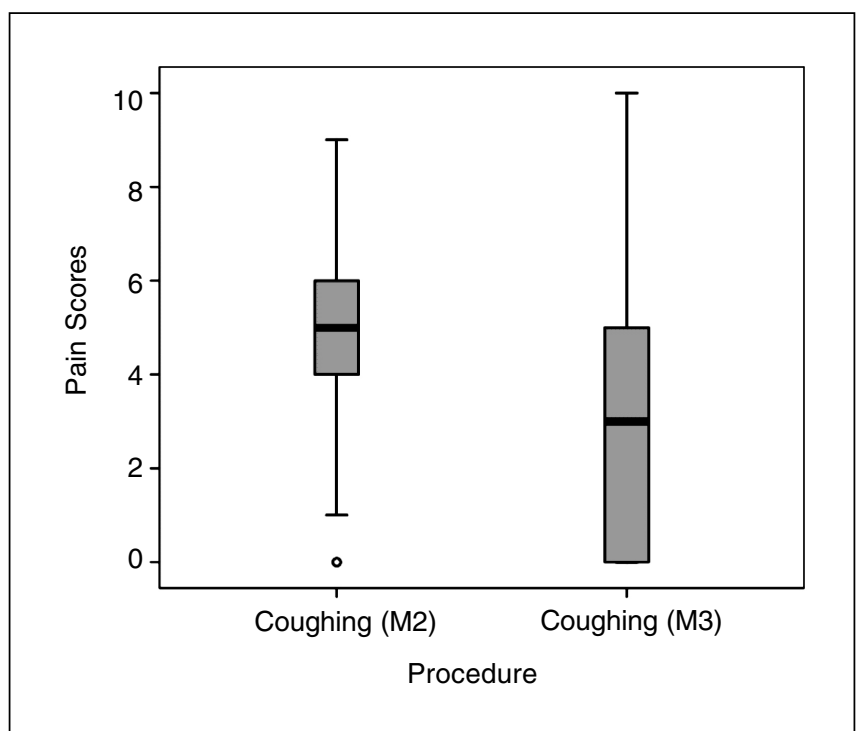

Figure 4 - Pain Scores among Moments and Procedures in the Study Group. There was a statistically significant difference in pain scores between M2 and M3 for coughing $(p=0.003)$, which was decreased after electrical stimulation and physical therapy (M3).

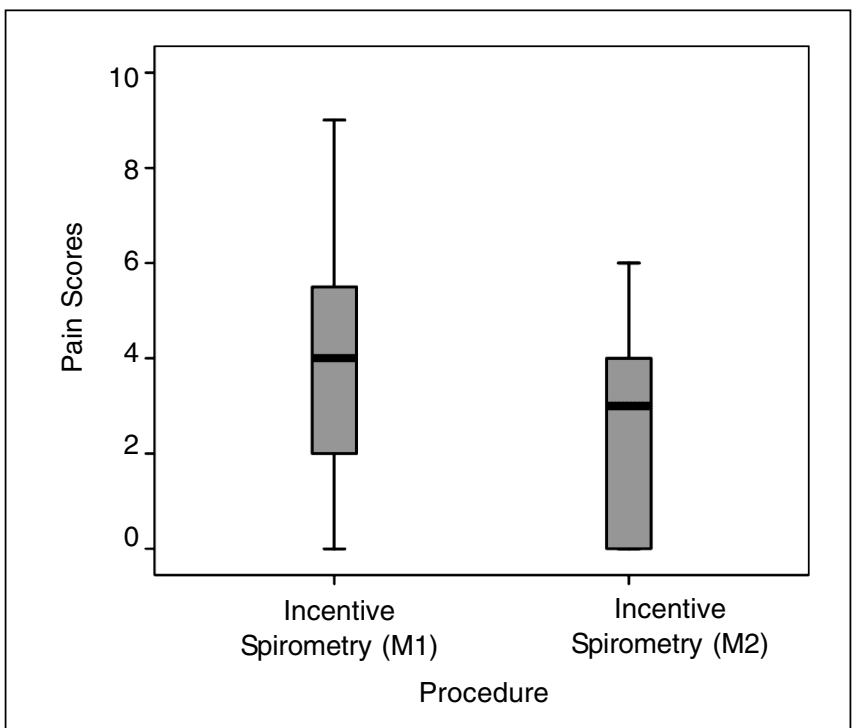

Figure 5 - Pain Scores among Moments and Procedures in the Study Group. There was a statistically significant difference in pain scores between M1 and M2 for incentive spirometry $(p=0.017)$, which was decreased after electrical stimulation.

manifest themselves. It is very often difficult to control with conventional analgesia with opioiods ${ }^{13}$. In 2001, Nguyen et al. ${ }^{14}$ concluded that pain at rest and with patient mobilization caused by laparoscopic gastroplasty was much less severe than that caused by the traditional surgery, with reduced use of postoperative morphine $(p<0.001)$, which was probably due to a reduced degree of surgical damage. In the current 
study, most patients complained of pain in the surgical incision in the immediate postoperative period.

The routine postoperative drug therapy for pain was maintained in every patient in this study. However, some of them were assisted by the physical therapist and evaluated by the protocol before the administration of the analgesics for that time. Statistical analysis showed that in all three groups the analgesic medication did not influence most of the procedures and moments.

Postoperative physical therapy is necessary for patient's recovery, having a preventive and curative action in some possible complications, such as atelectasis and penumonia ${ }^{1}$. Routine physical therapy procedures can increase the patient's pain ${ }^{15}$ because it uses manual resources and specific exercises associated with intense mobilization, improving respiratory function (maintenance of pulmonary permeability and expansion), and stimulating early ambulation (motor rehabilitation). Postoperative respiratory physical therapy includes manual and mechanical (using an electrical vibrator) maneuvers of pulmonary reexpansion, mobilization of secretions through manual thoracic vibro-compression, stimulation of cough by verbal commands, assisted coughing, stimulation of the sternal notch, and stimulation of pulmonary reexpansion through respiratory exercises and incentive spirometry ${ }^{16}$. Forced coughing, especially after surgeries in the upper abdomen, is one of the most painful procedures because it demands vigorous contraction of the diaphragm and abdominal musculature in the presence of a surgical wound with obvious inflammatory and painful processes. Although coughing is one of the procedures that increases pain the most, it is fundamental to mobilize and eliminate accumulated pulmonary secretions, guaranteeing the protection, hygiene, and equilibrium of the respiratory tract decreasing, therefore, the risk of atelectasis and infections.

Transcutaneous electrical nerve stimulation, a non-invasive procedure virtually devoid of side effects, can be used as an adjunct to conventional analgesia ${ }^{4,7,8,13}$. In the present study, TENS was chosen to promote postoperative pain relief, especially that secondary to physical therapy maneuvers, in patients who underwent abdominal surgeries because the greater the patient's cooperation and participation in the exercises, the better the efficacy of the technique and the faster his/her recovery and rehabilitation.

Recently, Bjordal et al ${ }^{10}$ published a metanalysis of the studies that used TENS for postoperative analgesia between 1966 and 2001. Results showed that TENS reduced the use of analgesics in the first three postoperative days, decreasing the side effects of opioids, besides promoting a statistically significant reduction in pain scores in the Study Group when compared to the Contrast-Placebo Group, which was also demonstrated in the present study. They also showed that it is necessary to use high intensity electrical currents (the maximum tolerated by the patient without causing discomfort) for better efficacy, which is also demonstrated in the present study.
Postoperative pain is caused, basically, by tissue damage and the resulting inflammatory process. There is also the primary postoperative hyperalgesia, which is an extension of the local painful process to areas adjacent to the lesion due to the increased release of painful mediators. This process reduces pain threshold and increases the sensitivity of adjacent nerve endings, causing hyperalgesia to stimuli that were not previously painful, such as touch ${ }^{17}$. Performing any procedure during this process can cause even more pain, especially while mobilizing and moving the patient, which reflects in the area of the surgical wound ${ }^{13}$.

In this study, TENS was effective in relieving pain caused by physical therapy maneuvers only for the parameter cough in $M 3$, i.e., after physical therapy $(p=0.015)$. It is important to decrease cough related pain due to the possible pulmonary complications that can happen after abdominal surgeries. Besides, decreasing pain when the patient coughs may guarantee an effective bronchial toilette, early pulmonary rehabilitation, and prevention of atelectasis and pneumonia secondary to accumulation of secretion. This data is similar to that obtained by Ali et al. ${ }^{9}$, who also demonstrated the efficacy of TENS in relieving postoperative pain when taking into consideration the increased forced vital capacity (FVC) and residual volume (RV), therefore decreasing the tendency for respiratory complications by decreasing pain in the surgical wound.

In the TENS Group there was also a reduction in pain scores when performing some procedures before and after electrical stimulation and after physical therapy, indicating the therapeutic importance of TENS as an adjuvant in respiratory and motor physical therapy.

Pain scores associated with coughing were reduced in the Study Group comparing scores between M1 and M3 $(p=0.009)$ and between $M 2$ and $M 3(p=0.003)$. This reduction in scores demonstrated the efficacy of TENS as an adjuvant in controlling pain for physical therapy. It is important to mention that the scores in M3 (after physical therapy) were even smaller than in M1 and M2. The efficacy of the analgesia with TENS was important, allowing patients to perform the necessary physical therapy procedures, and the reduction in the score at the last moment demonstrates the continuation of the analgesic effect of TENS during coughing. This effect obtained after physical therapy can be explained by the modulation of pain caused by TENS in the A delta fibers that conduct nociceptive stimuli. During mobilization, pain is transmitted through the A delta fibers. However, opioids inhibit the $C$ fibers, making it less effective in controlling pain triggered by moving or coughing ${ }^{12,13}$. Transcutaneous electrical stimulation generates a tactile stimulus that is transmitted by $\mathrm{C}$ fibers, closing the gate to the painful stimulus generated by the A delta fibers ${ }^{13}$.

Besides respiratory physical therapy, patients were also submitted to active exercises of the upper and lower limbs, sitting on a chair with assistance and, even after all these procedures, the final score when coughing maneuvers were performed did not increase. 
Incentive spirometry in the postoperative period of abdominal surgeries is recommended as an adjuvant in lung reexpansion, since it provides the patients feedback on their efforts through the number of spheres elevated with each inspiration and is widely used in hospitals to prevent pulmonary complications ${ }^{18}$. The proper use of this instrument depends on the understanding of the technique. A diaphragmatic reeducation is necessary to perform this exercise in order to promote pulmonary reexpansion, preferentially in the bases, with a predominantly diaphragmatic respiratory pattern and sustained maximal inspiration ${ }^{19}$. Diaphragmatic excursion is weakened by the painful process and local edema caused by the abdominal surgery, incentive spirometry is a potential source of added pain.

There was a significant reduction in pain scores with incentive spirometry in the Study Group when M1 was compared to M2. Transcutaneous electrical neuro stimulation reduced pain effectively for this parameter only in M2, i.e., shortly after electrical stimulation. Pain scores were not reduced in $M 3$, after physical therapy, and the analgesic efficacy of TENS in controlling pain associated with this parameter in M3 was not demonstrated. Since the knowledge about the technique can influence directly the performance of incentive spirometry, it is possible that the patients already knew the device and/or had a better respiratory pattern after physical therapy, while sitting outside the bed, which enabled them to use the device more effectively, resulting in a higher pain score than recorded shortly after TENS.

In the Study Group, the reduction in pain scores in the three moments also happened for decubitus change and sitting. There were statistical differences between $M 1$ and $M 2(p=$ $0.025)$ and between $M 1$ and $M 3(p=0.03)$ for decubitus change, and between $M 1$ and $M 2(p=0.001)$ and $M 1$ and $M 3$ $(p=0.001)$ for sitting. Note that the effectiveness of TENS in these procedures was already evident in $\mathrm{M} 2$, i.e., immediately after it was done. Its effects lasted until after physical therapy (respiratory and motor), with a lower score than the initial one (before TENS). The reduced score is important for decubitus change and sitting, since these procedures demand intense recruitment of the abdominal musculature (rectus abdominis and abdominal oblique), and this must be done in the presence of a surgical wound in the abdomen that is painful when mobilized ${ }^{13}$. Early mobilization is important because it prevents ileus ${ }^{4}$, the respiratory complications described previously, and even vascular complications, such as deep venous thrombosis ${ }^{20}$.

Ambulation was initially chosen as a parameter to evaluate pain scores, but its implementation was jeopardized because it had to be done in three moments, before any other intervention. Ambulation is usually done after all respiratory and motor physical therapy procedures in bed are done, precluding the inclusion of ambulation in the study, although it had been done during the routine motor physical therapy, in M3. Another point that is worth mentioning is that there are very few studies in the literature evaluating TENS as a means of decreasing pain during physical therapy. Most works compare analgesia with TENS and conventional analgesia, or the decreased need for analgesics with TENS without taking into consideration that patients need postoperative physical therapy. In 2003, Rakel and Frantz ${ }^{13}$ showed that TENS reduced the severity of pain during deep inspiration and ambulation in the first postoperative day.

Transcutaneous electrical neuro stimulation decreased pain scores for some procedures and moments. However, these results must be analyzed cautiously because the study population was small and the differences were not so noticeable. Thus, the use of TENS as a non-pharmacologic analgesic alternative should be further studied, since it is only indicated as an adjuvant in the control of postoperative pain and pain caused by physical therapy maneuvers.

\section{ACKNOWLEDGEMENTS}

The authors would like to thank the physical therapist Lucian Ysayama, master in Experimental Research by the Surgery Service of the Faculdade de Ciências Médicas da Universidade Estadual de Campinas (FCM-UNICAMP), and Professor José Milton Sanches for the statistical analysis of the data.

\section{REFERÊNCIAS - REFERENCES}

01. Imle PC - Fisioterapia em Pacientes com Problemas Cardíacos, Torácicos ou Abdominais após Cirurgia ou Trauma, em: Irwin SI, Tecklin JS - Fisioterapia Cardiopulmonar, $3^{\text {a }}$ ed., São Paulo, Manole, 2003;375-403.

02. Saad IAB, Zambom $L$ - Variáveis clínicas de risco pré-operatório. Rev Ass Med Bras, 2001;47:117-124.

03. White PF - Management of postoperative pain and emesis. Can J Anesth, 1995;42:1053-1055.

04. Marin LI, Castro CES - Estimulação elétrica nervosa transcutânea no controle da dor pós-laparotomia. Estudo preliminar. Rev Bras Anestesiol, 1986;36:207-214.

05. Melzack R, Wall P - Pain mechanisms: a new theory. Science, 1965;150:971-979.

06. Robinson AJ, Snyder-Mackler L - Eletrofisiologia Clínica Eletroterapia e Teste Eletrofisiológico, 2 ${ }^{\underline{a}}$ ed., Porto Alegre, Artmed, 2002;195-242.

07. Schomburg FL, Carter-Baker SA - Transcutaneous electrical nerve stimulation for postlaparotomy pain. Phys Ther, 1983; 63:188-193.

08. Benedetti F, Amanzio M, Casadio C et al - Control of postorative pain by transcutaneous electrical nerve stimulation after thoracic operations. Ann Thorac Surg, 1997;63:773-776.

09. Ali J, Yaffe CS, Serrette C - The effect of transcutaneous electric nerve stimulation on postoperative pain and pulmonary function. Surgery, 1981;89:507-512.

10. Bjordal JM, Johnson MI, Ljunggreen AE - Transcutaneous electrical nerve stimulation (tens) can reduce postoperative analgesic consumption. A meta-analysis with assessment of optimal treatment parameters for postoperative pain. Eur j pain, 2003;7:181-188.

11. Ledergerber CP - Postoperative electroanalgesia. Obstet Gynecol, 1978;51:334-337.

12. Ridley SC - Cirurgia em Adultos, em: Pryor JA,Webber BA - Fisioterapia para Problemas Respiratórios e Cardíacos, $2^{a}$ ed., Rio de Janeiro, Guanabara Koogan, 2002;210-233. 
13. Rakel B, Frantz R - Effectiveness of transcutaneous electrical nerve stimulation on postoperative pain with movement. J Pain, 2003;4:455-464.

14. Nguyen NT, Lee SL, Goldman C et al - Comparison of pulmonary function and postoperative pain after laparoscopic versus open gastric bypass: a randomized trial. J Am Coll Surg, 2001; 192:469-477.

15. Hall JC, Tarala RA, Tapper $\mathrm{J}$ et al - Prevention of respiratory complications after abdominal surgery: a randomised clinical trial. BMJ, 1996;312:148-153.

16. Costa $D$ - Manobras manuais de fisioterapia respiratória. Fisioterapia em Movimento, 1991;4:11-25.

17. Maquez JO - Bases de Anatomia e Fisiopatologia. Dor - Diagnóstico e Tratamento, 2004;1:1-8.

18. Lie $\mathrm{C}$, Kehlet $\mathrm{H}$, Rosemberg J - Lung physiotherapy as prophylaxis against atelectasis and pneumonia after abdominal surgery. Ugeskr Laeger, 1998;160:3540-3544.

19. Weindler J, Kiefer RT - The efficacy of postoperative incentive spirometry is influenced by the device-specific imposed work of breathing. Chest, 2001;119:1858-1864.

20. Eisele R, Kinzl L - Prevention of thrombosis with physical therapy in trauma surgery. Possibilities and value of individual measures. Unfallchirug, 1998;101:851-855.

\section{RESUMEN}

Tonella RM, Araújo S, Silva AMO - Estimulación Eléctrica Nerviosa Transcutánea no Alivio del Dolor Postoperatorio Relacionado con los Procedimientos Fisioterapéuticos en Pacientes Sometidos a Intervenciones Quirúrgicas Abdominales.

JUSTIFICATIVA Y OBJETIVOS: LA electroestimulación nerviosa transcutánea (TENS) ha sido poco estudiada como método facilitador de la fisioterapia postoperatoria. El objetivo del estudio fue el de verificar la eficacia de la TENS en el alivio del dolor relacionado a la fisioterapia postoperatoria en pacientes sometidos a intervenciones quirúrgicas abdominales.

MÉTODO: Se realizó un estudio clínico, prospectivo, con distribución aleatoria, incluyendo 48 pacientes en el 1ํo día de postoperatorio, que presentaban un puntaje de dolor $\geq 3$ puntos en la escala visual analógica (VAS), divididos en tres grupos: Grupo Control: con rutina analgésica habitual, sin TENS y con fisioterapia; Grupo Estudio, recibiendo rutina analgésica habitual más TENS, y fisioterapia; Grupo Contraste-Placebo, recibiendo rutina analgésica habitual, fisioterapia y TENS desvinculada. Se presentó a los pacientes una escala visual analógica de dolor antes (M1), después de la TENS (M2) y después de la fisioterapia (M3) - tos, incentivador respiratorio, cambio de decúbito lateral y sentado - cuantificando la efectividad de la analgesia. El tiempo de electroestimulación fue de 30 minutos.

RESULTADOS: Hubo un alivio significativo del dolor en el Grupo Estudio, comparándolo con los tres grupos, apenas para el procedimiento tos, en el M3 $(p=0,015)$. Dentro de ese grupo hubo una disminución significativa del dolor para tos $(p=0,003)$ [M1 versus M3]; para decúbito lateral $(p=0,025)$, sentarse $(p=0,001)$ y utilizar el incentivador inspiratorio ( $p=0,017)$ [M1 versus M2]; $y$ al cambiar para decúbito lateral $(p=0,03)$ y sentarse $(p=0,001)$ [M1xM3]. No hobo diferencia significativa en el Grupo Contraste Placebo.

CONCLUSIONES: Hubo disminución del dolor en el Grupo Estudio, en algunos momentos y parámetros. Estudios adicionales se hacen necesarios, ya que la utilización de la TENS está indicada apenas como coadyuvante en el control del dolor postoperatorio. 